\title{
Image-guided minimally invasive percutaneous treatment of spinal metastasis
}

\author{
PING-LIN YANG, XI-JING HE, HAO-PENG LI, QUAN-JIN ZANG and GUO-YU WANG \\ Second Department of Orthopedics, The Second Affiliated Hospital of Xi'an Jiaotong University, \\ Xi'an, Shaanxi 710004, P.R. China
}

Received March 10, 2015; Accepted May 11, 2016

DOI: $10.3892 /$ etm.2017.4029

\begin{abstract}
In order to provide effective options for minimally invasive treatment of spinal metastases, the present study retrospectively evaluated the efficacy and safety of image-guided minimally invasive percutaneous treatment of spinal metastases. Image-guided percutaneous vertebral body enhancement, radiofrequency ablation (RFA) and tumor debulking combined with other methods to strengthen the vertebrae were applied dependent on the indications. Percutaneous vertebroplasty (PVP) was used when vertebral body destruction was simple. In addition, RFA was used in cases where pure spinal epidural soft tissue mass or accessories (spinous process, vertebral plate and vertebral pedicle) were destroyed, but vertebral integrity and stability existed. Tumor debulking (also known as limited RFA) combined with vertebral augmentation were used in cases presenting destruction of the epidural soft tissue mass and accessories, and pathological vertebral fractures. A comprehensive assessment was performed through a standardized questionnaire and indicators including biomechanical stability of the spine, quality of life, neurological status and tumor progression status were assessed during the 6 weeks- 6 months follow-up following surgery. After the most suitable treatment was used, the biomechanical stability of the spine was increased, the pain caused by spinal metastases within 6 weeks was significantly reduced, while the daily activities and quality of life were improved. The mean progression-free survival of tumors was $330 \pm 54$ days, and no associated complications occurred. Therefore, the use of a combination of image-guided PVP, RFA and other methods is safe and effective for the treatment of spinal metastases.
\end{abstract}

Correspondence to: Dr Xi-Jing He, Second Department of Orthopedics, The Second Affiliated Hospital of Xi'an Jiaotong University, 157 West Fifth Road, Xi'an, Shaanxi 710004, P.R. China E-mail: xijinghe1@163.com

Key words: minimally invasive spinal surgery, spinal metastasis, vertebroplasty, radiofrequency ablation, image guidance

\section{Introduction}

The spine is a common site of metastasis in cancer patients. Spinal metastasis is observed in $60-70 \%$ of patients with systemic cancer; the frequency with which spine metastases are detected varies considerably with the type of primary tumor (1). Common tumors with a high rate of metastasis to bone include tumors of the breast (72\%), prostate (84\%), thyroid (50\%), lung (31\%), kidney (37\%), and pancreas (33\%) (1). The incidence of spinal metastases is increasing due to early detection and advances in the treatment of the primary tumour. In the United States alone, $>350,000$ cases of spinal metastasis are reported each year due to prostate, breast, kidney, lung, and thyroid cancers (2). Due to a more profound understanding of the disease and higher quality of life requirements, an increasing number of patients undergo surgery for the treatment of spinal metastasis (3). Surgical intervention methods, including multi-channel minimally invasive treatment under radiological guidance, are currently commonly used in the treatment of the disease (4). The curative effect of spinal metastasis treatment by radiofrequency ablation (RFA) (5), percutaneous vertebroplasty (PVP) $(6,7)$ or a combination of PVP and RFA has greatly improved in recent years, as also observed at the Second Affiliated Hospital of Xi'an Jiaotong University Medical School (Xi'an, China). Real-time image-guided RFA is a minimally invasive treatment recently developed for treating vertebral tumors, using imageguided positioning of an electrode in the lesion to kill malignant cells percutaneously with minimal damage to adjacent healthy tissues (8). Augmentation through the injection of a reinforcing biomaterial (PVP), is a popular, minimally invasive intervention for the stabilization of fractured vertebral bodies (9). Through the use of these therapies, pain resulting from osteolytic bone metastases of the spine is relieved immediately, while the spinal strength and stability are increased, vertebral collapse is prevented, the activity status of patients is improved and local tumor cell death is increased.

In the present study, the clinical data of patients treated with a combination of PVP and RFA were retrospectively analyzed.

\section{Patients and methods}

Patients. Clinical data of spinal metastasis patients treated between June 2010 and June 2013 at the Second Affiliated Hospital of Xi'an Jiaotong University Hospital were obtained. 
This retrospective analysis was approved by the Ethics Committee of the Second Affiliated Hospital of Xi'an Jiaotong University Hospital. A total of 42 patients participated in the tumor progression survey and subsequent 2-year follow-up. Among the 42 spinal vertebral metastases cases (involving 52 vertebrae), 25 patients (32 vertebrae) were treated with RFA in combination with PVP (group A). A total of 17 patients ( 20 vertebrae) were treated with PVP alone (group B). In group A, some patients presented with destruction of the epidural soft tissue mass and accessories, and pathological vertebral fractures; these patients were treated with tumor debulking (limited RFA) in combination with PVP. The patient cohort included 32 males and 10 females, with an age range of 26-88 years (mean age, 63 years). The involved vertebrae included 21 thoracic-lumbar, 19 lumbar, 5 cervical and 7 sacral vertebrae. Spinal metastasis was observed in patients with various primary tumors, including 12 cases of lung cancer, 4 cases of breast carcinoma, 7 cases of colon carcinoma, 6 cases of renal cell carcinoma, 1 case of gastric cancer, 2 cases of liver cancer, 8 cases of prostate cancer, and 2 cases of thyroid carcinoma.

Eligible candidates for the study met the following conditions: i) Evident history of the primary tumor, or diagnosis by aspiration biopsy; ii) experienced sudden or persistent pain in the neck, chest, back or waist; and iii) complete clinical record available. Follow-up was performed by a combination of telephone and out-patient appointments.

Basic characteristics of the patients treated with RFA combined with PVP were collected prior to surgery and are shown in Table I. Karnofsky index scores were determined for all patients (as percentages), based on the following evaluation criteria (10): $100 \%$, normal activity, no complaints and no evidence of disease; $90 \%$, able to perform normal activities, minor signs or symptoms of disease; $80 \%$, normal activity with some effort required, and some signs and symptoms of disease; $70 \%$, cares for self, but unable to perform normal activities or work; $60 \%$, occasionally requires assistance, but can care for the majority of personal needs; $50 \%$, requires considerable assistance and frequent medical care; $40 \%$, disabled patient, requiring special care and assistance; $30 \%$, severe disability, requires hospital admission, but vital signs were stable; $20 \%$, highly severe disability, requiring hospitalization and active supportive treatment; $10 \%$, moribund, with rapid progression of fatal processes; $0 \%$, mortality. In addition, the Pain Disability Index score was determined, which indicates the degree to which the daily lives of patients are disrupted by chronic pain ( 0 , no effect; 10 , maximum effect) (11). The PDI was determined by investigation of seven categories, including the ability to perform family responsibilities, recreation, social activities, occupation, sexual behavior, self-care ability and life-support activities.

Surgical materials and equipment. Simplex P bone cement (also known as polymethylmethacrylate) was obtained from Stryker Corp. (Mahwah, NJ, USA). For PVP, Murphy Coaxial-M2 bone access needles (gauge, 11 and 13) were purchased from Cook Medical Inc. (Bloomington, IN, USA). Hi-Visco Flow cement pressurized syringe and pasteurized barium sulfate were purchased from Heraeus Medical GmbH (Wehrheim, Germany). Siemens Axiom Artis dFA
Table I. Basic characteristics of the patients treated with RFA combined PVP.

\begin{tabular}{lc}
\hline Variable & Value \\
\hline Age, years & $62.7 \pm 9.0(46-82)$ \\
Number of affected vertebrae & $1.8 \pm 1.1(1-6)$ \\
Karnofsky index, $\%$ & $65.9 \pm 14.3(60-90)$ \\
PDI & \\
Family responsibilities & $5.8 \pm 3.4(0-10)$ \\
Recreation & $6.4 \pm 3.5(0-10)$ \\
Social activities & $5.6 \pm 3.5(0-10)$ \\
Occupation & $6.0 \pm 3.8(0-10)$ \\
Sexual behavior & $7.2 \pm 3.3(0-10)$ \\
Self-care ability & $5.0 \pm 3.7(0-10)$ \\
Life-support activities & $2.8 \pm 2.8(0-10)$
\end{tabular}

Data are presented as the mean \pm standard deviation, with the range in parenthesis. The PDI indicates the degree to which the daily lives of patients are disrupted by chronic pain ( 0 , no effect; 10 , maximum effect). RFA, radiofrequency ablation; PDI, pain disability index.

angiography system along with X-Leonardo VA60B work stations were obtained from Siemens Healthcare (Erlangen, Germany).

Preoperative preparation. Preoperative assessments of cardiopulmonary function and blood coagulation function were performed, which involved analyzing the number of lesions in the spine, the size of the lesion site, soft tissue mass and infiltration, spinal cord compression and spinal stability. Lesion locations and size were determined by computed tomography (CT) and magnetic resonance imaging in all the patients. CT was performed using a Lightspeed VCT-XT (GE Healthcare Bio-Sciences, Pittsburgh, PA, USA). MRI was performed using Magnetom Avanto 1.5T (Siemens AG, Munich, Germany). The preoperative imaging data of lesions and adjacent tissue were analyzed. Care was taken to mimic the puncture route of surgery before the procedure. Phenobarbital sedation (2\% lidocaine; 200-400 mg; Shanghai Sym Pharma Co., Ltd., Shanghai, China) was performed $30 \mathrm{~min}$ before surgery.

Interventional surgery method. Using digital subtraction angiography (DSA) guidance with a Siemens Axion Artis dFA angiography, the vertebral lesions were identified and marked to select an appropriate puncture route. Surgery divided into the following steps: i) Puncture: Lidocaine anesthesia (2\%) was performed and the patient was punctured layer by layer to the pedicle periosteal and the bone cortex. A puncture was performed into the vertebral lesions with an 11-G or 13-G needle (Osteosite Bone Biopsy Needle Murphy M2; Cook, Bloomington, IN, USA) the transfixion pin method, according to CT simulation of the puncture route; ii) RFA: The pin core was pulled out of the lesion and a radiofrequency ( $R F$ ) electrode needle was injected into the target location under fluoroscopy. The gauge size of the 
needle was selected according to the tumor size, and the needle was then connected to the RF signal generator; and iii) PVP injection of bone cement: The RF pin was removed, the pin core was inserted into the needle cannula, and then the needle was inserted up to $1 / 3$ of the anterior vertebral body under fluoroscopy. The pin core was pulled out, and barium sulfate mixed with polymethylmethacrylate cement (Simplex P; Stryker Orthopaedics, Mahwah, NJ, USA) was injected slowly using Hi Visco Flow bone cement pressurized syringe. When Visco Flow bone cement pressurized syringe slowly, the entire injection process was monitored by DSA until the bone cement distribution was homogeneous.

Hematoxylin and eosin staining. Following CT scanning, the puncture plane was determined according to scan images and the most obvious pathological lesions were selected. Under local anesthesia, a puncture needle was inserted via the predetermined path to reach the predetermined depth and position. Once the puncture position was confirmed, the targeted tissue was harvested 2 to 3 times from different directions. The tissue samples were frozen and subsequently subjected to hematoxylin and eosin staining.'

Visual analog scale (VAS). Pain intensity was determined according to the VAS scores of patients (12). The commonly used approach involves the use of a 0-10 scale, where ' 0 ' indicates no pain and ' 10 ' stands for highly intense pain. Patients were instructed to mark the corresponding position on the rule that represented their degree of pain. In the present study, patients were followed up until mortality or the end of the follow-up period, and VAS scores were recorded immediately and 1, 3, 6, and 12 months after RFA + PVP surgery. The mean follow-up duration was 7.8 months.

Frankel classification. The Frankel classification of patients was analyzed at 2, 6 and $>12$ months after surgery, as previously described (13).

Statistical analysis. Results were analyzed using SPSS version 15.0 software (SPSS, Inc., Chicago, IL, USA) for statistical analysis. Parametric data were expressed as mean \pm standard deviation and were compared using Student's t-tests. P-values were generated using Wilcoxon signed-rank test for comparisons of the pain at each time point with those prior to surgery. Differences with $\mathrm{P}<0.05$ were considered as statistically significant.

\section{Results}

Surgical results. All the patients successfully underwent surgery under DSA guidance. RFA of vertebral body was first performed, during which the temperature was maintained at $50-100^{\circ} \mathrm{C}$ continuously for $5-15 \mathrm{~min}$, and the amount of bone cement injected was 2-7 ml. The surgical success rate of RFA was $100 \%$, with no skin burns, blood vessel or nerve damage, and other complications observed during RFA. During PVP surgery, vertebral disc leakage occurred in 1 case, paravertebral leakage in 3 cases and cement leakage in 2 cases. In addition, 1 patient presented spinal cord compression symptoms, and the condition was improved symptomatically

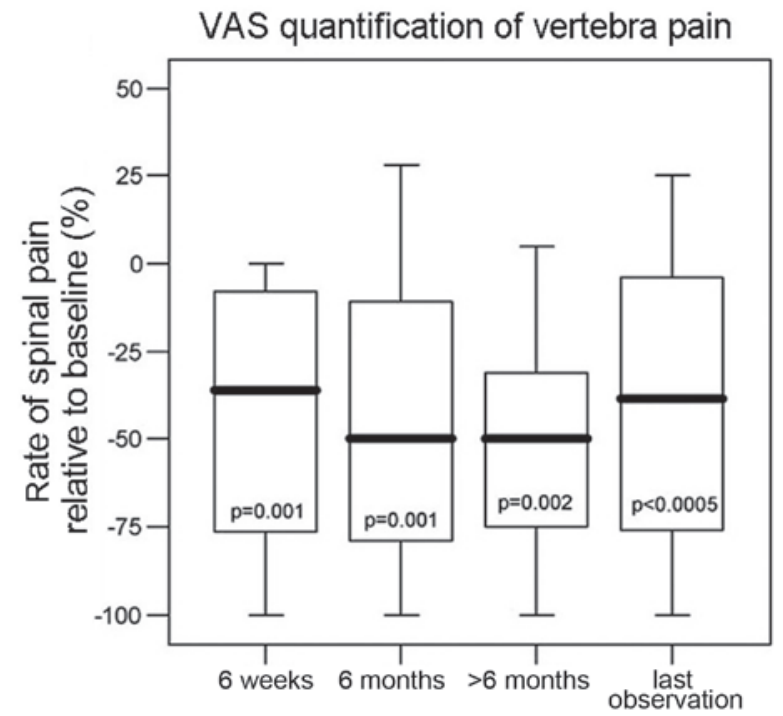

Figure 1. Spinal pain VAS scores (relative to the baseline) at various time points after surgery. P-values were generated using Wilcoxon signed-rank test for comparisons of the pain at each time point with that prior to surgery. VAS scores were determined based on a $0-100 \mathrm{~mm}$ ruler $(0 \mathrm{~mm}$ indicated no pain and maximum pain was $100 \mathrm{~mm}$ ). VAS, visual analog scale.

after 7 days with the use of hormones and dehydration. Upon retrospective review of the data, the 2 cases of spinal leakage occurred due to spinal vertebral bone destruction, and the integrity of the vertebral structure was closely associated with the complications.

Evident pyknosis was observed in the tumors following RFA using CT imaging, and the mean intraoperative blood loss was $524.5 \mathrm{ml}$. No mortality cases or cases of postoperative paralysis were observed. The duration of follow-up was $>6$ months in all cases (range, 7-15 months; mean follow-up, 7.8 months). Effective postoperative pain was relieved, with the exception of 1 patient with primary thyroid cancer patients experiencing local recurrence after 8 months. Furthermore, 2 patients of lung cancer metastasis succumbed to the disease at 7 and 8 months after surgery, since the primary tumor was not controlled successfully.

Pain relief. Pain relief resulting from spinal tumor reduction when compared with the baseline assessment value (prior to surgery) is shown in Fig. 1 using VAS measurements. Due incomplete information, the number of patients was different at each time point of the pain evaluation. Compared with the baseline values, the VAS values and thus the intensity of pain were significantly reduced after surgery.

Frankel classification. Neurological status was evaluated via the Frankel classification of patients analyzed at 2,6 and $>12$ months after surgery, based on the following evaluation criteria: A, complete injury (paraplegia); B, only sensory function; C, motor function present, but no practical use (non-amputation); D, motor function present, ability to walk (outpatients); and E, no neurological signs or symptoms. Patients who maintained or regained a status equivalent to Frankel type E or D were considered good responders. Patients who were nonambulatory after treatment were considered nonresponders by this criterion. One patient was paraplegic 
Table II. Frankel classification of patients at different follow-up times.

\begin{tabular}{lllllr}
$\begin{array}{l}\text { Time after } \\
\text { surgery }\end{array}$ & A & B & C & D & E \\
\hline 2 months & 0 & 1 & 5 & 23 & 13 \\
6 months & 0 & 2 & 6 & 21 & 11 \\
$>12$ months & 1 & 2 & 8 & 20 & 8 \\
\hline
\end{tabular}

A, complete injury (paraplegia); B, only sensory function; $\mathrm{C}$, motor function present, but no practical use (non-amputation); D, motor function present, ability to walk (outpatient); E, no neurological signs or symptoms.

prior to surgery and at 1 year after surgery among all the follow-up patients. Furthermore, $>70 \%$ of patients (28/39) were able to walk and presented improved motor function after surgery. The Frankel classification data are shown in Table II.

Although no complications occurred, 4 patients reported side effects associated with RFA. Of these, as a side effect of RFA, 2 patients experienced contralateral lower limb pain and numbness during the procedure; however, the side effect abated when the temperature decreased subsequent to RFA. In addition, 2 patients received high doses of corticosteroids after RFA. Furthermore, 1 patient experienced heaviness in the legs 1 day after RFA and the symptom subsided within 1 week.

Tumor progression. A total of 42 patients participated in the tumor progression survey and subsequent 2-year follow-up. During the 2-year follow-up, 28 of the 42 patients (66.67\%) did not present tumor progression. However, 5 patients showed partial tumor progression, with the tumors penetrating into the nerve segments. These patients were retreated with reduced-energy RFA (energy output, $<50 \mathrm{~W}$ ) under local anesthesia. Normally, the typical RFA energy output for paravertebral tumor is $>80 \mathrm{~W}$ within $15 \mathrm{~min}$. During the follow-up period, the tumors exhibited variable progression rates with a mean progression-free survival of $330 \pm 54$ days.

\section{Discussion}

RFA is a technology of coagulated necrosis that uses a radio wave $(450-500 \mathrm{kHz})$ to vibrate electrodes inserted into the lesions and heat the surrounding tissue $\left(50-100^{\circ} \mathrm{C}\right)(14)$. Due to the high temperature applied during this technique, controlled intervention and establishing the exact location of lesions are crucial, particularly in high-risk sites such as the spine. Previous studies have demonstrated that a probe placed inside the bone is less likely to cause thermal damage of neural structures $(5,15)$. Although the bone tissue appears to have an insulating function and reduce heat transfer, injury to the surrounding tissue, such as muscles, can occur when high heat is applied (16). There are several possible risks in RFA, including bleeding, infection, injury of adjacent structures and abscesses (17,18).

The PVP surgical procedure is performed by percutaneous puncture causing a small and rapid trauma; however, PVP is a palliative treatment aimed at relieving pain within a limited time (19). It is suitable for older and weak patients with poor tolerance to surgery, but with intact posterior of vertebral and cortical pedicle. PVP can stabilize the fracture, prevent pathological fracture and deformity, and strengthen the spinal stability (20).

The combination of RFA and PVP for the treatment of malignant tumors of the spine can effectively enhance the strength and hardness of the spine, while causing the death of tumor cells $(21,22)$. Selection of the appropriate RF needle position, radio temperature and procedure duration is significant to prevent complications in the RFA treatment of spinal metastases. For the selections of the appropriate parameters, the following must be performed: First, the tumor size and location must be evaluated preoperatively by $\mathrm{CT}$ in order to select the appropriate electrode needle to cover the tumor as much as possible. The distance of the electrode needle from important organs, large blood vessels or nerves should be $1 \mathrm{~cm}$ or more (4) to ensure safety. In addition, the temperature used during RFA should be appropriately reduced if the vertebral bone cortex was damaged $(23,24)$. In the present study, the target temperature was set to $60-75^{\circ} \mathrm{C}$, and no RF induced nerve and spinal cord injury was observed. Although there was cortical destruction of the vertebral bone, the temperature range was safe and feasible. Tumor necrosis contributes to the distribution of bone cement. In the current study, the pathology of specimens obtained from patients prior to and following RFA was investigated. Prior to RFA, a large number of tumor cells and blood vessels was observed in pathological images. Subsequent to surgery, the number of pathologically visible tumor cells was reduced, while a small amount of blood vessels and numerous necrotic tissues were observed.

RFA as a method to kill tumor cells has been used in the clinical treatment of bone and soft tissue tumors $(25,26)$. In recent years, an increasing number of scholars attempted to use the RFA treatment technique for spinal tumors. There is a certain risk that the spinal cord is injured during percutaneous RFA treatment of spinal tumors. A number of reports using RFA treatment for spinal tumors demonstrated cancer pain relief, and indicated that approach is minimally invasive compared to surgery, since the spinal nerves are protected by constant cooling via rinsing during the RFA process. Tumor size, vascular occlusion and bleeding during the removal of the tumor were significantly reduced subsequent to RFA $(5,27)$. Although complete removal of the tumor is not guaranteed by surgical treatment and RFA, the combination of these treatments can improve the extent and safety of the tumor removal $(28,29)$.

RFA is a safe, economical, efficient transdermal technology in the treatment of patients with primary and secondary tumors, as well as unresectable tumors in the spinal canal and those that are insensitive to chemotherapy and radiotherapy $(30,31)$. It can prevent or delay tumor progression and the risk of fracture, thus preventing or delaying paraplegia, reducing the pain caused by the tumor, and improving the daily activities and life quality of patients.

In conclusion, RFA combined with PVP for the treatment of spinal metastasis is able to quickly relieve pain, kill tumor cells, increase the vertebral strength and improve the stability of the spine. RFA followed by PVP can reduce the risk of leakage of bone cement, particularly the leakage in vascular extrapyramidal 
or dura regions, while it can also improve the safety of PVP treatment. Due to spinal metastasis being a systemic disease, there are certain disadvantages of RFA followed by PVP as a topical treatment. Considering the type of the primary tumor, the treatment should be selected according to the individual case. Furthermore, the combination of PVP with various drugs, radiotherapy and further surgical therapy should also be considered in future investigations.

\section{References}

1. Shah LM and Salzman KL: Imaging of spinal metastatic disease. Int J Surg Oncol 2011: 769753, 2011.

2. Mundy GR: Metastasis to bone: Causes, consequences and therapeutic opportunities. Nat Rev Cancer 2: 584-593, 2002.

3. Arrigo RT, Kalanithi P, Cheng I, Alamin T, Carragee EJ, Mindea SA, Park J and Boakye M: Predictors of survival after surgical treatment of spinal metastasis. Neurosurgery 68: 674-681; discussion 681, 2011

4. Coronel EE, Lien RJ and Ortiz AO: Postoperative spine imaging in cancer patients. Neuroimaging Clin N Am 24: 327-335, 2014

5. Nakatsuka A, Yamakado K, Takaki H, Uraki J, Makita M, Oshima F and Takeda K: Percutaneous radiofrequency ablation of painful spinal tumors adjacent to the spinal cord with real-time monitoring of spinal canal temperature: A prospective study. Cardiovasc Intervent Radiol 32: 70-75, 2009

6. Sun G, Li L, Jin P, Liu XW and Li M: Percutaneous vertebroplasty for painful spinal metastasis with epidural encroachment. J Surg Oncol 110: 123-128, 2014.

7. Mendel E, Bourekas E, Gerszten P and Golan JD: Percutaneous techniques in the treatment of spine tumors: What are the diagnostic and therapeutic indications and outcomes? Spine (Phila Pa 1976) 34 (Suppl 22): S93-S100, 2009.

8. Goetz MP, Callstrom MR, Charboneau JW, Farrell MA, Maus TP, Welch TJ, Wong GY, Sloan JA, Novotny PJ, Petersen IA, et al: Percutaneous image-guided radiofrequency ablation of painful metastases involving bone: A multicenter study. J Clin Oncol 22: 300-306, 2004.

9. Widmer Soyka, RP, et al., The effectiveness of percutaneous vertebroplasty is determined by the patient-specific bone condition and the treatment strategy. PLoS One 11: e0151680, 2016.

10. Chambless LB, Kistka HM, Parker SL, Hassam-Malani L, McGirt MJ and Thompson RC: The relative value of postoperative versus preoperative Karnofsky Performance Scale scores as a predictor of survival after surgical resection of glioblastoma multiforme. Neuro Oncol 121: 359-364, 2015.

11. Tait RC, Pollard CA, Margolis RB, Duckro PN and Krause SJ: The Pain Disability Index: Psychometric and validity data. Arch Phys Med Rehabil 68: 438-441, 1987.

12. Nakatsuka A, Yamakado K, Maeda M, Yasuda M, Akeboshi M, Takaki H, Hamada A and Takeda K: Radiofrequency ablation combined with bone cement injection for the treatment of bone malignancies. J Vasc Interv Radiol 15: 707-712, 2004.

13. Bollen L, van der Linden Y M, Pondaag W, Fiocco M, Pattynama BP, Marijnen CA, Nelissen RG, Peul WC and Dijkstra PS: Prognostic factors associated with survival in patients with symptomatic spinal bone metastases: A retrospective cohort study of 1043 patients. Neuro Oncol 16: 991-998, 2014.

14. Sun CZ J: The clinical appliance of radiofrequency ablation. Journal of Interventional Radiology 16: 502-504, 2007.
15. McAlinden N, Massoubre D, Richardson E, Gu E, Sakata S, Dawson MD and Mathieson K: Thermal and optical characterization of micro-LED probes for in vivo optogenetic neural stimulation. Opt Lett 38: 992-994, 2013.

16. Earhart J, Wellman D, Donaldson J, Chesterton J, King E and Janicki JA: Radiofrequency ablation in the treatment of osteoid osteoma: Results and complications. Pediatr Radiol 43: 814-819, 2013.

17. Dupuy DE, Hong R, Oliver B and Goldberg SN: Radiofrequency ablation of spinal tumors: Temperature distribution in the spinal canal. AJR Am J Roentgenol 175: 1263-1266, 2000.

18. Goldberg SN, Solbiati L, Halpern EF and Gazelle GS: Variables affecting proper system grounding for radiofrequency ablation in an animal model. J Vasc Interv Radiol 11: 1069-1075, 2000.

19. Masala S, Anselmetti GC, Muto M, Mammucari M, Volpi T and Simonetti G: Percutaneous vertebroplasty relieves pain in metastatic cervical fractures. Clin Orthop Relat Res 469: 715-722, 2011.

20. Schaefer O, Lohrmann C, Herling M, Uhrmeister P and Langer M: Combined radiofrequency thermal ablation and percutaneous cementoplasty treatment of a pathologic fracture. J Vasc Interv Radiol 13: 1047-1050, 2002.

21. Morassi LG, Kokkinis K, Evangelopoulos DS, Karargyris O, Vlachou I, Kalokairinou K and Pneumaticos SG: Percutaneous radiofrequency ablation of spinal osteoid osteoma under CT guidance. $\mathrm{Br}$ J Radiol 87: 20140003, 2014.

22. Song HM, Gu YF, Li YD, Wu CG, Sun ZK and He CJ: Interventional tumor removal: A new technique for malignant spinal tumor and malignant vertebral compression fractures without epidural involvement. Acta Radiol 55: 976-984, 2014.

23. Thiagalingam A, D'Avila A, McPherson C, Malchano Z, Ruskin J and Reddy VY: Impedance and temperature monitoring improve the safety of closedloop irrigated-tip radiofrequency ablation. J Cardiovasc Electrophysiol 18: 318-325, 2007.

24. Iyer V, Gambhir A, Desai SP, Garan H and Whang W: Successful simultaneous unipolar radiofrequency ablation of septal ventricular tachycardia using 2 ablation catheters. Heart Rhythm 11: 710, 2014.

25. Lanuti M, Sharma A, Digumarthy SR, Wright CD, Donahue DM, Wain JC, Mathisen DJ and Shepard JA: Radiofrequency ablation for treatment of medically inoperable stage I non-small cell lung cancer. J Thorac Cardiovasc Surg 137: 160-166, 2009.

26. Filippiadis DK, Tutton S, Mazioti A and Kelekis A: Percutaneous image-guided ablation of bone and soft tissue tumours: A review of available techniques and protective measures. Insights Imaging 5: 339-346, 2014

27. Tian QH, Wu CG, Gu YF, He CJ and Li MH: Combination radiofrequency ablation and percutaneous osteoplasty for palliative treatment of painful extraspinal bone metastasis: A single-center experience. J Vasc Interv Radiol 25: 1094-1100, 2014

28. Kushchayeva YS, Kushchayev SV, Wexler JA, Carroll NM, Preul MC, Teytelboym OM, Sonntag VK, Van Nostrand D, Burman KD and Boyle LM: Current treatment modalities for spinal metastases secondary to thyroid carcinoma. Thyroid 24: 1443-1455, 2014.

29. Aboulafia AJ, Levine AM, Schmidt D and Aboulafia D: Surgical therapy of bone metastases. Semin Oncol 34: 206-214, 2007.

30. Desolneux G, Vara J, Razafindratsira T, Isambert M, Brouste V, McKelvie-Sebileau P and Evrard S: Patterns of complications following intraoperative radiofrequency ablation for liver metastases. HPB (Oxford) 16: 1002-1008, 2014.

31. Lee JM, Jin GY, Goldberg SN, Lee YC, Chung GH, Han YM, Lee SY and Kim CS: Percutaneous radiofrequency ablation for inoperable non-small cell lung cancer and metastases: Preliminary report. Radiology 230: 125-134, 2004. 